\title{
Thermomechanical couplings in aircraft tire rolling/sliding modeling
}

\author{
A. Kongo Kondé ${ }^{1,2}$, I. Rosu², F.Lebon ${ }^{2}$, L. Seguin ${ }^{2}$ O.Brardo ${ }^{1}$, F.Troude ${ }^{1}$, \\ B.Devésa ${ }^{1}$
}

\author{
${ }^{1}$ AIRBUS OPERATIONS S.A.S, 316 route de Bayonne, 31060 Toulouse Cedex 03 \\ ${ }^{2}$ LMA-CNRS, 31 Chemin Joseph-Aiguier, 13402 Marseille Cedex 20 \\ ange.kongo-konde@airbus.com, kongo@Ima.cnrs-mrs.fr, rosu@lma.cnrs-mrs.fr, \\ lebon@lam.cnrs-mrs.fr, olivier.brardo@airbus.com, bernard.devesa@airbus.com, \\ florian.troude@airbus.com
}

Keywords: Aircraft tire, Finite element, Friction law, Rubber, Steady State Rolling, Temperature.

\begin{abstract}
This paper presents a finite element model for the simulation of aircraft tire rolling. Large deformations, material incompressibility, heterogeneities of the material, unilateral contact with Coulomb friction law are taken into account. The numerical model will allow estimating the forces in the contact patch - even in critical and extreme conditions for the aircraft safety and security. We show the influence of loading parameters (vertical load, velocity, inflating pressure) and slip angle on the Self Aligning torque and on the lateral friction coefficient. A friction coefficient law corresponding to Chichinadze model is considered to take into account thermal effects in the aircraft tire model behaviour.
\end{abstract}

\section{Introduction}

Landing and take-off operations are the critical phases of a flight. Indeed, the high speed, the violent impact when aircraft touches the ground, the need of braking efficiently, the ground irregularities, the climatic conditions (crosswind, contaminations, ...) as well as other parameters can make these maneuvers on ground very perilous.

Knowing at each time the forces in the contact patch could allow mastering the behaviour in the tire/ground interface. For aeronautical applications, the experiment on real tire, at moderated speeds, yields to knowledge of tire/ground behaviour in this reduced field. However, the high nonlinearities related to materials, geometry due to large deformations, coupled phenomena (contact with friction, thermal-mechanical coupling, wear) reduce the trust in extrapolation capabilities of these results at high speeds.

When we study tire yawed rolling, it is relevant to well estimate the Self Aligning Torque and the ratio, lateral force/ normal force. These respectively express the ability of tire to return in its steering direction when it is no longer steered, and the lateral grip potential of the rubber/ground couple.

Indeed, the ratio $\mu_{\mathrm{Y}}$ (between lateral force and vertical one) defines the equilibrium which exists between steering lock action and the trajectory of the vehicle. During $\mu_{\mathrm{Y}}$ upward phase, there is equilibrium, the more the driver or the pilot turns the wheel the more $\beta$ increases. It corresponds to pseudo-sliding in the contact patch up to $\beta_{\max }$ (around $\beta=4$ to $7^{\circ}$ for automobile pneumatics, around $\beta=6$ to $10^{\circ}$ for trucks and around 15 to $25^{\circ}$ for aircraft, see Fig. 1).

Beyond these values, a loss of equilibrium sets up: the more the driver tries to increase the drift, the more the vehicle slides. This phase corresponds to pure sliding in the contact. The rubber is warming up, the temperature increases and implies decreasing of the friction coefficient $\mu$. Thus, we observe the decreasing of $\mu_{Y}$ and $\mathrm{M}_{Z}$ as shown in Fig. 1. The specific values are normalized for analysing the observed phenomena. 


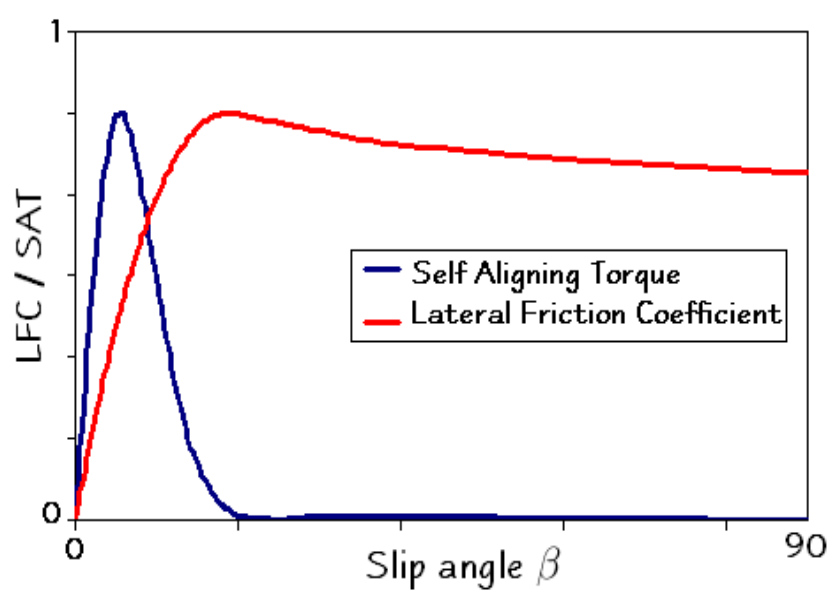

Fig. 1: Self - Aligning Torque and Lateral Friction coefficient for aircraft tire.

In skid, the result of friction forces is at the rear of the contact area center. This intrinsic property of rubber creates resistant torque which faces drift: this is the Self - Aligning Torque, $\mathrm{M}_{\mathrm{Z}}$. It represents the pneumatic ability to return to his steering direction when the driver releases vehicle controls. It is maximal for slip angle $\beta=6^{\circ}$ and rapidly vanishes towards zero (around $\beta=18^{\circ}$ for aircraft tire), see Fig. 1.

Numerical modelling using Finite Element Analysis and the current computer capabilities open the way to produce finer and finer simulations of industrial problems formerly limited in terms of degrees of freedom. This new way allows also reducing the operating cost of experiments.

\section{Aim of the study}

The aim of this work is to highlight the effects of loading parameters (vertical load, velocity, inflating pressure) on the mechanical behaviour of tire in contact with the ground. In extreme conditions (crab landing due to crosswind for example), the high energy dissipation in the contact zone results in an increasing of temperature and wear. The local friction coefficient $\left(\mu_{\text {local }}\right)$ depends, a priori, highly on temperature, wear, sliding velocity and contact pressure. To emphasize the effects of these variations on the global mechanical behaviour of $\mu_{Y}$ and $\mathrm{M}_{Z}$, we must consider a thermal-mechanical coupling. To do that, we propose a thermal model of tire in which the aim is to define the evolution of temperature during tire rolling and we also focus on the temperature diffusion in its thickness.

\section{Finite Element Model for Tire Analysis FEA Model}

Finite Element Analysis (FEA) is used nowadays to predict behaviour and performance of structures. Regarding our study, an accurate 3D tire model is essential. The fundamental requires of such modelling process are the accuracy and adequate information of the tire cross-section [1], particularly the internal features of plies and their modelling. The simulation process includes these following features:

- Tire geometry: Some image acquisition technologies developed for inspection operations in the tire manufacturing process are now being adapted by researchers for acquiring tire geometric data for relevant studies in the absence of any CAD tire geometry data [2]. This method provides the 2D section of tire including all tire inner features. So, the tire data are then stored in appropriate format and imported into ABAQUS software for further FE Modeling. Suitable points are then chosen as the nodes before including them into FE input file. 


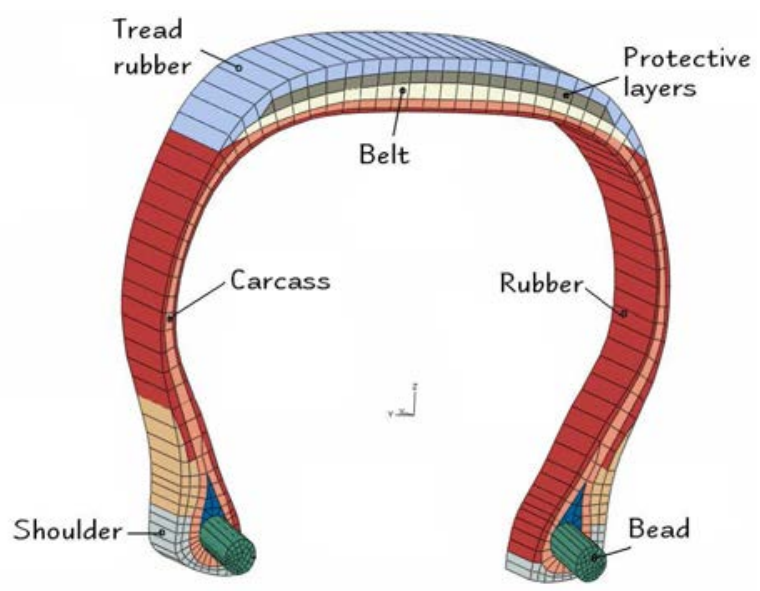

Fig. 2: 2D-section and 3D-partial models.

- Rubber and Reinforced fiber behaviours: The hyperelastic model of Mooney Rivlin [3] is largely used to model the rubber behaviour while orthotropic elastic model defines the reinforced fiber behaviour. The material properties considered in this study are derived from experimental tension test performed on samples cut in each different layers of the tire (see Fig. 2). We assume that the dependence of mechanical characteristics on temperature is neglected.

- Tire/rim contact: The contact of the tire to the rim is simplified with assumption that tire sticks to the rim. The nodes at the tire/rim contact are fixed at the rim by the tie option in ABAQUS.

- Coulomb friction at tire/ground contact: The tire/ground contact complicates the finite element model, since contact and friction problems are highly non-linear. Nonetheless, both effects cannot be ignored. The contact problem in FEM model is described by a deformable body (tire) to rigid body (ground) option and modeled by soft contact using penalty regularization. The Coulomb friction is modeled by a stiffness method.

Table 1: Mechanical assumptions.

\begin{tabular}{|c|c|c|}
\hline & Constitutive law & Dependence on Temperature \\
\hline \hline Rubber & Mooney Rivlin & No \\
Fibers & Orthotropic Elasticity & No \\
Tire / rim contact & Perfect adhesion & No \\
Tire / Ground & Signorini contact, Coulomb friction $\mu$ & Yes \\
\hline
\end{tabular}

The 3D finite element model is created in two steps. Firstly, the 2D mesh is created. Based on this mesh, 3D model is generated by revolution. Fig. 2 describes the FEM model. It shows the FE mesh of $2 \mathrm{D}$ model. It consists on 4-nodes elements and some 3-nodes required for the modeling of geometrical transition between the tread and sidewall and between sidewall and shoulder.

These 3- and 4-nodes elements are transformed, respectively, in 3D elements with 6- and 8-nodes. The reinforced layers are modeled using in the same way. Fig. 2 shows a partial 3D mesh model. It consists on 21863 elements, 43663 nodes and 120853 variables in the model. 


\section{Thermomechanical Coupling}

In grip phenomenon, two mechanisms take place under the influence of relative sliding between rubber and ground: indentation and adhesion. In both cases, rubber viscoelastic characteristics play an important role. Some studies on rubber samples show significant influence of contact pressure, of the temperature and of the sliding velocity on the friction coefficient. Generally, this friction coefficient reaches a maximum value around $90^{\circ} \mathrm{C}$ before decreasing with the increasing of temperature [4]. The temperature evolution in the contact patch is, thus, an important step which allows knowing the local friction coefficient $\mu_{\text {local }}$.

During a contact with friction between two bodies $(\Omega 1$ and $\Omega 2)$, there is generation of heat in the contact due to energy dissipation. Generally, to solve a thermal problem leads to finding the solution of the heat equation (Eq. 1) with boundary conditions: Dirichlet and Neumann (convection, adiabatic and given temperature).

$$
\rho . c_{p} \frac{d T}{d t}=\operatorname{div}(k \cdot \operatorname{grad} T)+Q
$$

Where $\rho$ is the mass density $\left(\mathrm{kg} / \mathrm{m}^{3}\right), c_{p}$ is the specific heat $\left(\mathrm{Jkg}^{-1} \mathrm{~K}^{-1}\right), k$ is the thermal conductivity $\left(\mathrm{Wm}^{-1} \mathrm{~K}^{-1}\right), T$ is the temperature $(\mathrm{K}), Q$ is the internal and external heat sources $\left(\mathrm{Wm}^{-3}\right)$ and $t$ is the time (s).

In our study, the heat equation and material characteristics are only applied on the elastic body and the dissipated flux due to friction $Q_{f}$ can be written as a function of $\alpha$. So, this flux is written on the contact boundary as:

$$
Q_{f}=\alpha \cdot \mu_{\text {local }}(T) \cdot \lambda_{N} \cdot \dot{u}_{T}
$$

With $\lambda_{N}$ contact pressure, $\dot{u}_{T}$ tangential speed in the contact, $\mu_{\text {local }}(T)$ the local friction coefficient depending on temperature, and $\alpha$ the share coefficient. We assume that material properties do not vary with the temperature. The friction coefficient only depends on the temperature.

\section{Methods and Analysis approaches}

Two approaches [5] can be used to resolve this kind of problem. The first one is called "steady state analysis". This is a mixed Lagrangian/Eulerian approach in which, for an observer in the reference frame, the tire is a fixed set while materials flow through the refined stationary mesh.

The second one is called "Classical Dynamic analysis". This Lagrangian approach allows simulating real rolling. In this approach, for an observer in the reference frame, the tires rolls and, turn after turn, the elements touch and leave the contact area.

We have already discussed about the advantages and disadvantages of the two strategies [5]. Numerically, both approaches are equivalents.

\section{Static Analysis}

The static analysis is performed in consecutive stages. In the first stage, the tire is modeled under inflation pressure using 2D axisymmetric model. Inflation analysis of axisymmetric model is followed by $3 \mathrm{D}$ static analysis of vertically loaded tire. The analysis consists of several steps. In this step, the 3D model is created, by rotation of an axisymmetric model around tire axis. In step one tire is inflated, while in step two ground surface gets gradually moved towards tire axis, until it reaches the goal value. In step three, equilibrium is reached, with constant force acting on surface. This step is needed as the basis for subsequent steady state rolling analysis or dynamic rolling analysis. 


\section{Steady State Analysis "SS"}

In this analysis, the tire was modeled under steady state rolling conditions $[6,7]$. This stage is restarted from the results of the second step (footprint analysis). A constant given ground velocity was assumed for the tire. The tire spinning velocity was changed to determine the tire free rolling condition and its dynamic loaded radius. In addition, the friction between tire tread surface and ground was taken into account using the simple linear Coulomb's law. The last analysis step is devoted to the application of the slip angle to the tire for a cornering situation. Slip angle is defined as the angle between the direction of travel and a normal plane to the axis of rotation. Analyses were performed using these values in conjunction with tire free rotational speed found in the previous step. The cornering is obtained by divided the rolling speed $v_{0}$ into $v_{x}=v_{0} \cos \beta$ and $\mathrm{v}_{\mathrm{y}}=\mathrm{v}_{0} \sin \beta$, where $\beta$ is the slip angle.

\section{Results}

\section{Static validation of numerical model}

This static analysis is primarily performed because of model integrity checked, finding the deformed shape of the model.

Fig. 3 shows the load-deflection curves from our tire model, manufacturer data and experimental results. The comparison reveals that there is a good matching between reference data (manufacturer data and experimental results) and computed values of tire model. So, what about the contact area?

We do not model the tread pattern features. So, the tire tread is smooth and without grooves, (see Fig. 2). The deletion of the tire features modifies the contact area and makes it greater. The increasing of contact area due to this observation is about $21 \%$ (see Table 2). Nevertheless, this value remains large. Further investigations have to be done to better match the contact area.

Table 2: Static validation of tire model.

\begin{tabular}{|c|c|c|c|}
\hline & Numerical & Experimental & Manufacturer \\
\hline \hline Contact area & 1.21 & -- & 1.0 \\
Vertical deflection & 1.05 & -- & 1.0 \\
Lateral deflection & 1.01 & 1.0 & -- \\
\hline
\end{tabular}
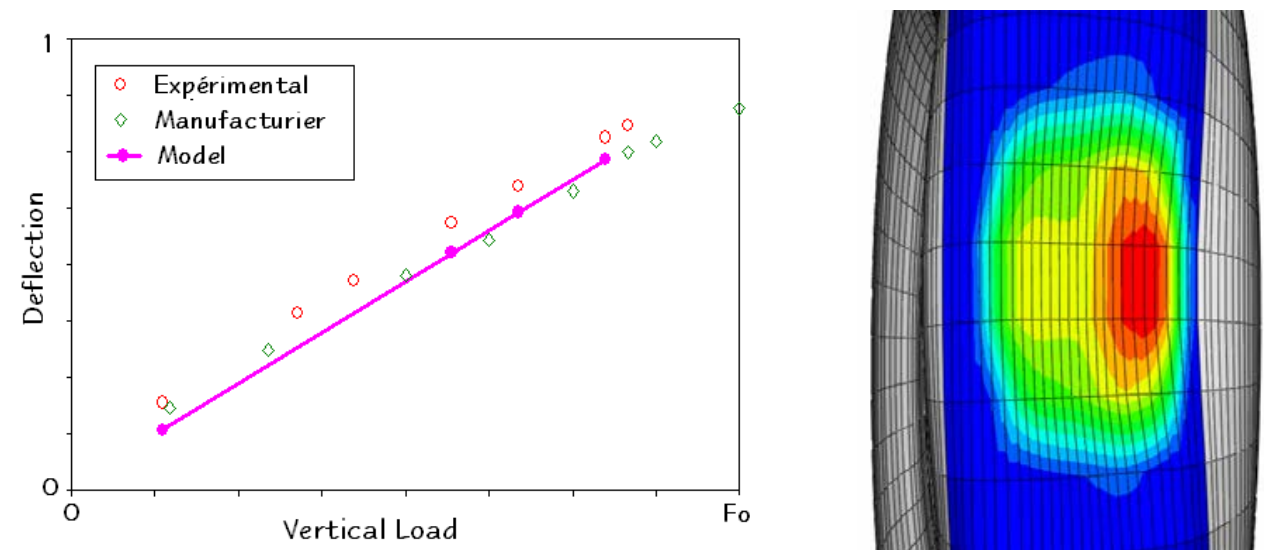

Fig. 3: Vertical deflection and contact area for $\beta=16^{\circ} \mathrm{C}$ 


\section{Simulation of tire rolling}

Simulations are carried for different rolling velocities $\left(v=0.6 v_{0}\right.$ to $\left.6 v_{0}\right)$, vertical loads $\left(F_{Z}=\right.$ $0.15 F_{0} ; 0.39 F_{0}$ and $\left.0.69 F_{0}\right)$, nominal inflating pressure $\left(P=P_{0}\right)$, slip angle $\left(\beta=16^{\circ}\right)$, constant friction coefficient $\mu_{0}$ and varying friction $\mu=\mu_{0} . f(T)$.

\section{Loading parameters effects}

- Rolling velocity effects: Fig. 4 shows the comparison between simulations performed at constant vertical load, pressure and friction coefficient by varying the rolling velocity. We observe that $\mu_{Y}$ is independent of rolling velocity, but there exists a threshold velocity at which the magnitude of $\mathrm{M}_{\mathrm{Z}}$ decreases exponentially with the growing speed. The value of $\beta_{\max }$ related to the maximum of $\mathrm{M}_{\mathrm{Z}}$ does not change. So, the high rolling velocities affect the magnitude of $\mathrm{M}_{\mathrm{Z}}$.
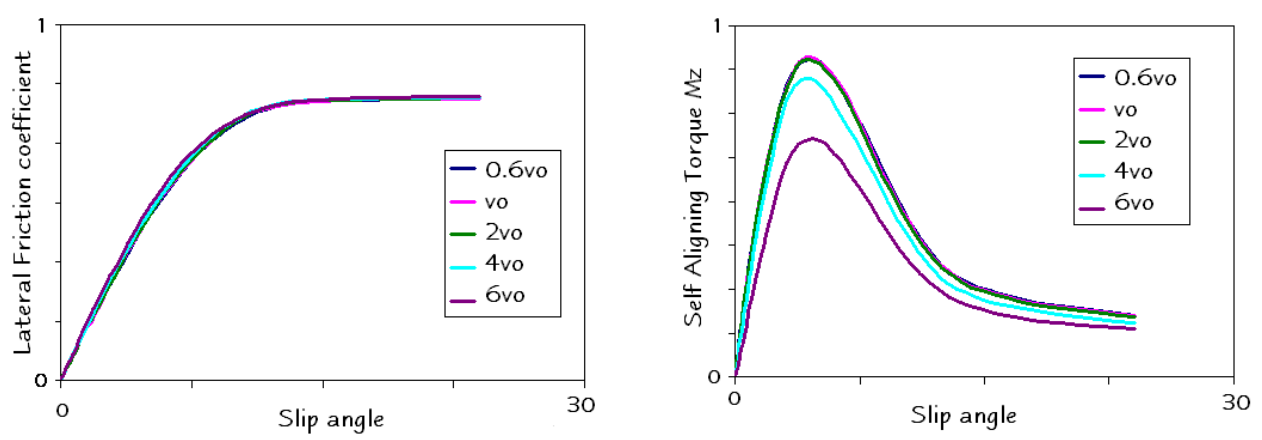

Fig. 4: Rolling velocity effects on $\mu_{\mathrm{Y}}$ and $\mathrm{M}_{\mathrm{Z}}$

- Vertical load effects: Fig. 5 shows the evolution of $\mu_{Y}$ and $\mathrm{M}_{Z}$ with the vertical load $\mathrm{F}_{Z}$. The influence of vertical load on the mechanical tire response is important. We observe that $\mu_{Y}$ is not sensitive to $F_{Z}$ while $\beta_{\max }$ increases. We also observe a sensitive increasing of $\mathrm{M}_{\mathrm{Z}}$ magnitude and a shift of $\beta_{\max }$ (Fig. 5) toward greater values while the vertical load increases. This observation was predictable. Indeed, the forces in the contact patch increase proportionately with the vertical load. De facto, $\beta_{\max }$ corresponding to the slip angle for which contact nodes slide, increases.
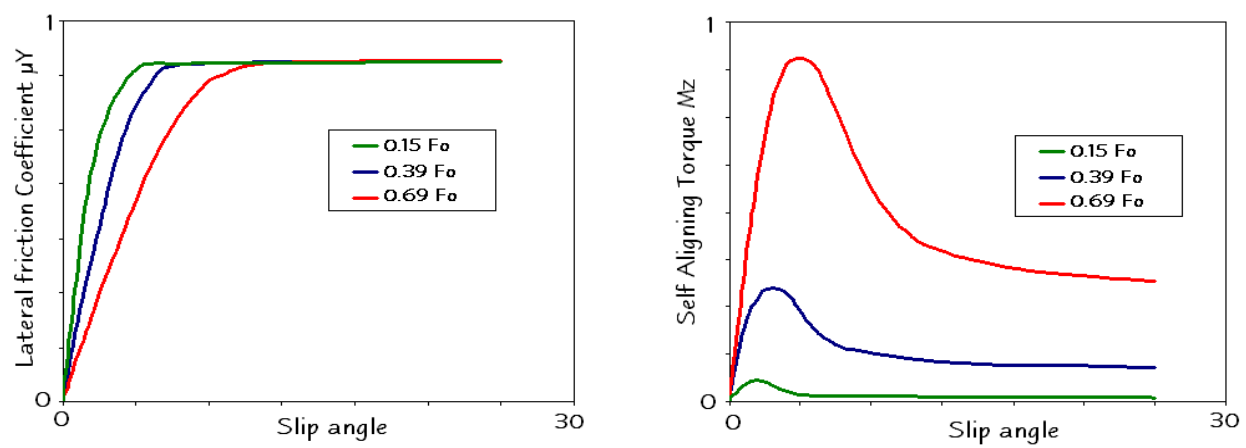

Fig. 5: Load effects on $\mu_{\mathrm{Y}}$ and $\mathrm{M}_{\mathrm{Z}}$ 
- Thermal - Mechanical Simulations: Thermal characteristics (conductivity, specific heat) are derived from [8]. The pure sliding (Fig. 6) is obtained by pulling the tire. We use the ABAQUS thermal - mechanical capabilities to performe the following simulations. The tire is pulled on ground along 4 meters at constant speed of $v_{0}, v_{0}$ is the reference velocity. In this case, we observe that the temperature increases significantly when the tire is lengthways slided down even on a short distance. The rubber reaches a superficial temperature of $190^{\circ} \mathrm{C}$ (Fig. 6) close to critical temperature for which the rubber is deposited on concrete road [9].
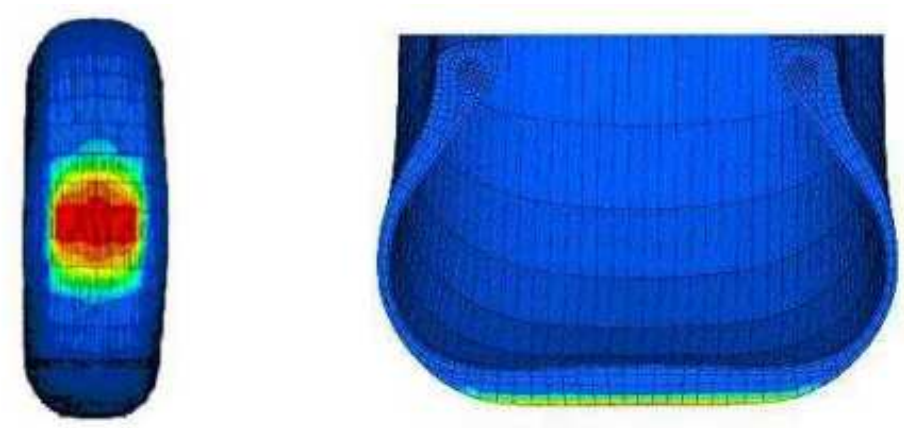

Fig. 6: Thermal result in pure sliding.

The running velocities considered are $v_{0}$ and $2 v_{0}$ at $\beta=16^{\circ}$. The Fig. 7 shows the temperature distribution of the tire running at $\mathrm{v}_{0}$. In every instance, the rise in temperature is located on tire tread surface with the temperature gradient within the thickness (see Fig. 7 a). In Fig. 7 b, we observe that the warming is in the sliding direction. The temperature is higher from right side to left side on tire tread surface. The reinforced zone seems to be a thermal barrier. However, the thermalmechanical analysis remains too expensive.
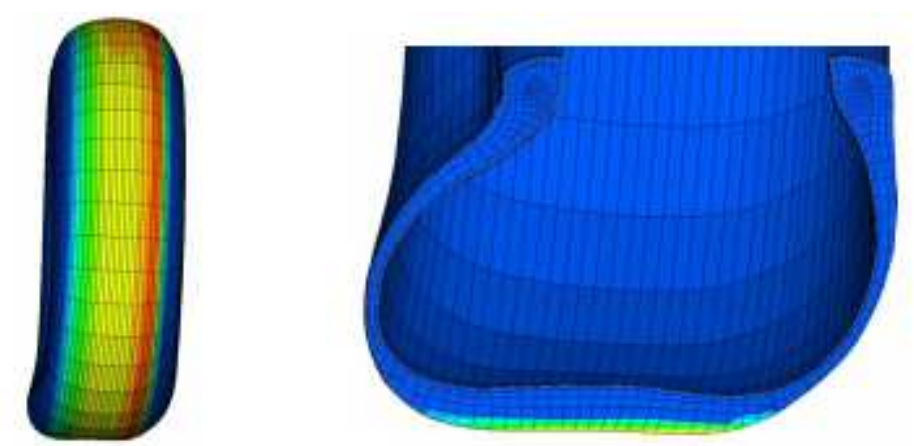

Fig. 7: Thermal result in cornering.

Fig. 8 shows the evolution of temperature of a tire tread node for the two velocities. This evolution is a step-like curve where the plateau represents the node temperature when it is out of the contact patch and the slope characterizes the increasing node temperature in the contact patch. When the node is out of the contact region, its temperature keeps constant because we do not consider the convective effects between tire and surrounding air. Normally, we should have a slight decrease due to convection which is a very complex phenomenon, and naturally depends on velocity $\mathrm{k}=\mathrm{f}(\mathrm{v})$.

The main conclusion of this analysis is that the temperature values depend on the speed at which the tire is rolling. 


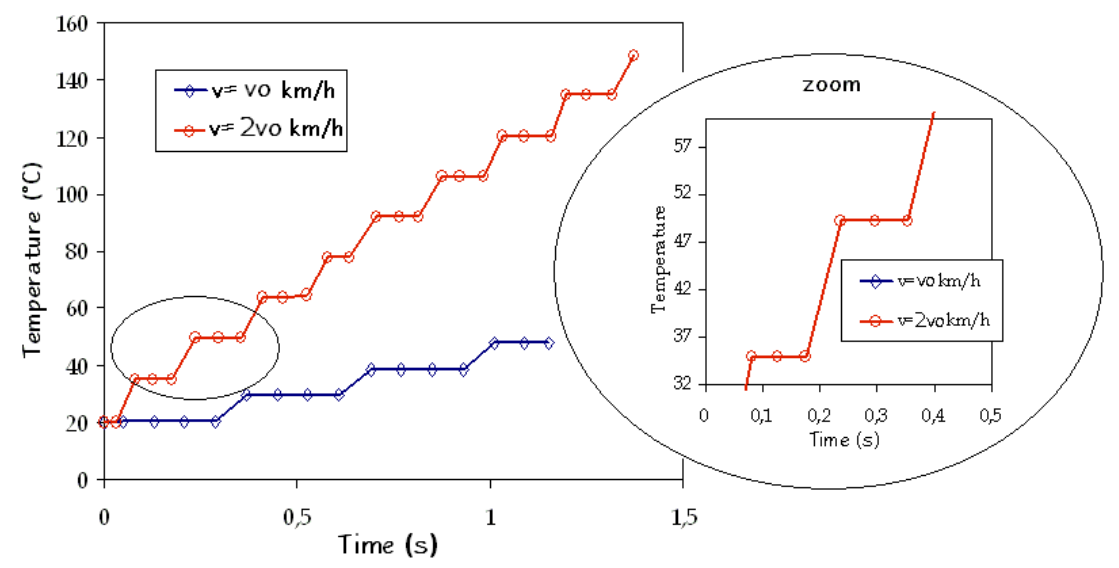

Fig. 8: Rise in temperature of a tire node at vo and 2 vo

- Effects of considering a variable friction coefficient: The warm-up to due friction in the contact patch has the effect of modifying the local friction coefficient. To take these effects into account in the modelling of the tire behaviour, we consider the following friction law (Eq. 3) [10] in which friction coefficient depends on temperature.

$$
\mu_{\text {local }}(T)=\mu_{0}\left(f_{1}+\frac{f_{2}}{f_{3}\left(T_{0}-T\right)^{2}+1}\right)
$$

We performed the above test rig (Fig. 10) inspired from Coulomb apparatus. Samples are cut in tread rubber and mounted on an aluminium block to ensure optimal contact between sample and the surface and to keep the sample at constant temperature.

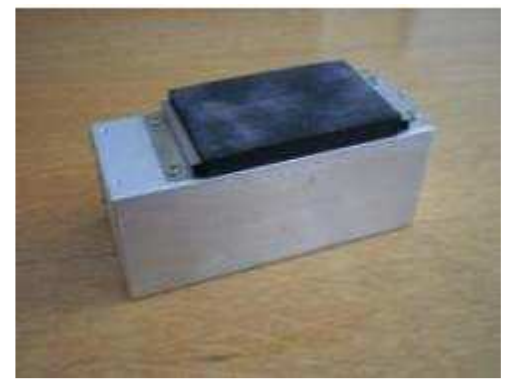

Fig. 9: Aluminium block and sample

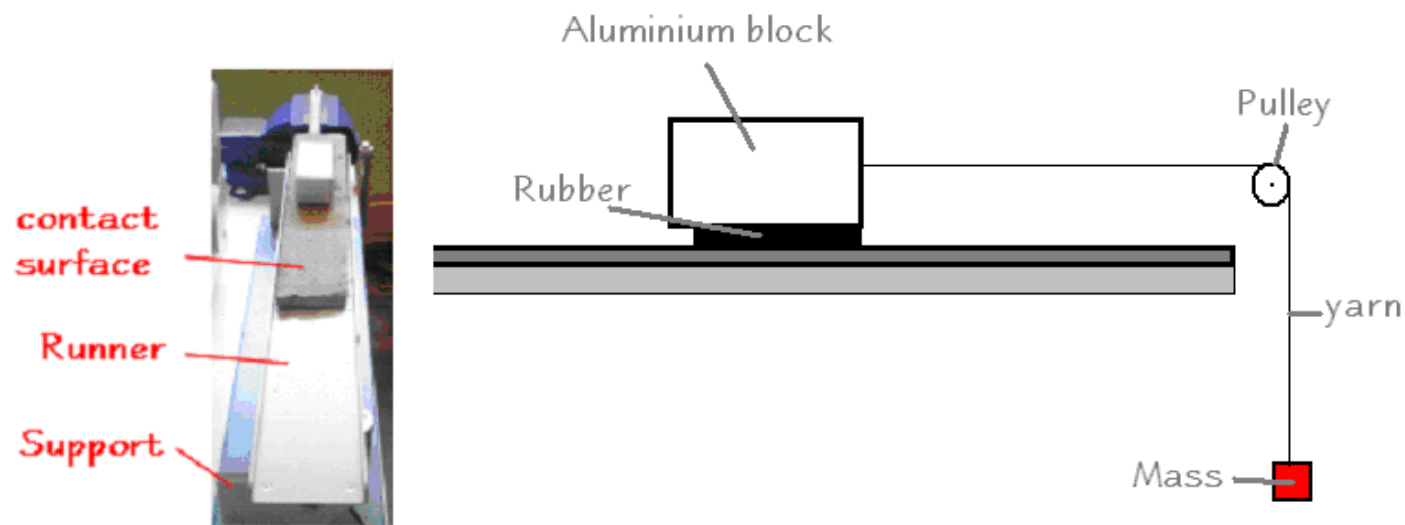

Fig. 10: Test device 
Fig. 9 shows aluminium block and sample. The friction coefficient was measured for concrete and asphalt surfaces. The results of these tests are presented on the Fig. 11.

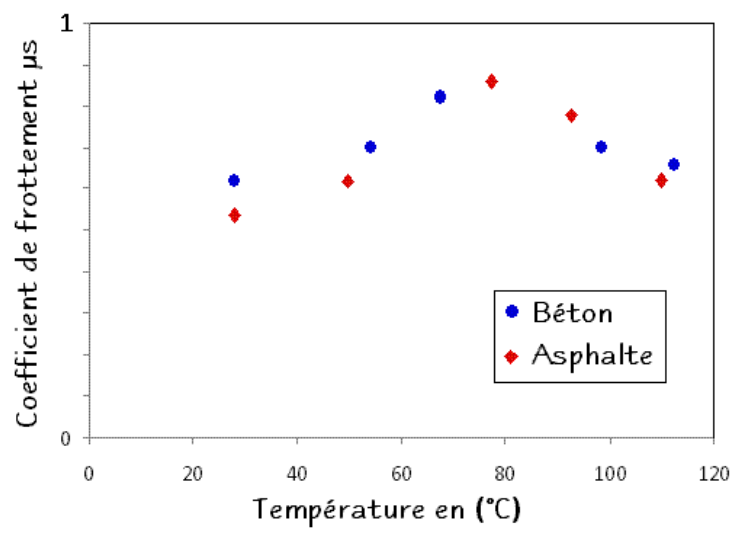

Fig. 11: Friction coefficient $\mu$ function of $\mathrm{T}$ and road surfaces

So, we can now identify $f_{i}$ variables of Chichinadze law (Eq. 3). As we expected, the dependence of $\mu$ on temperature induces a decrease of $\mu_{\mathrm{Y}}$ beyond $\beta_{\max }$ value and $\mathrm{M}_{\mathrm{Z}}$ tends rapidly toward zero [4]. Results are shown Fig. 12.
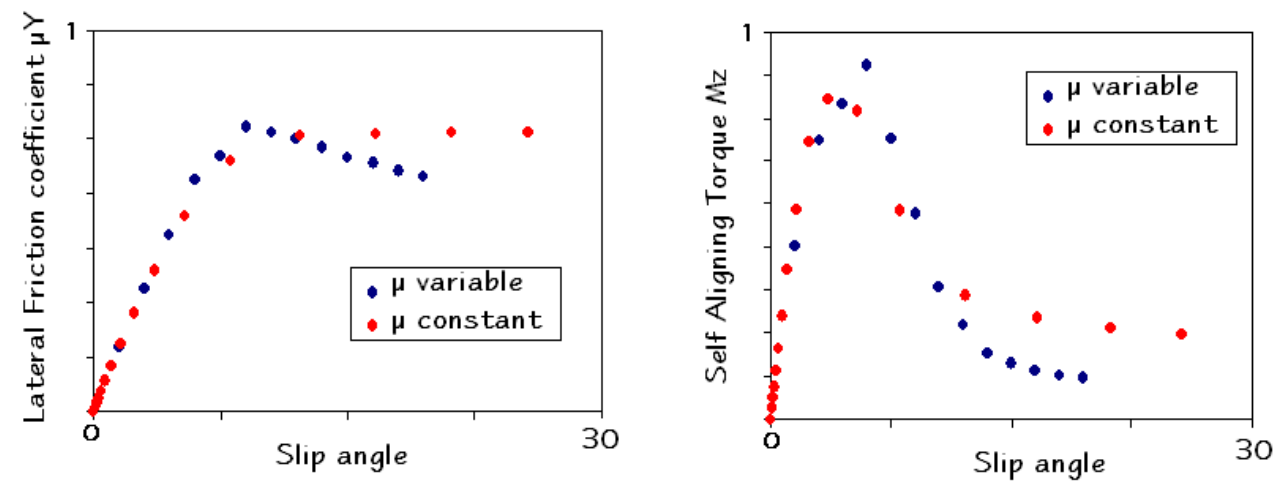

Fig. 12. Comparison of mechanical response with constant and varying friction coefficient (Chichinadze) on $\mu_{\mathrm{Y}}$ and $\mathrm{M}_{\mathrm{Z}}$

\section{Conclusion}

The present research is a first step on the modelling of aircraft tire in which thermal effects are described via a friction law depending only on temperature. A Coulomb-like test rig allows the measuring of the static friction coefficient of rubber/concrete and rubber/asphalt. Indeed, taking into account these effects in the friction coefficient, we emphasize the decreasing of $\mu_{\mathrm{Y}}$ and the rapid vanishing of $\mathrm{M}_{\mathrm{Z}}$ towards zero beyond $\beta_{\max }$ for which the full grip potential is reached as we did by considering, in first approximation, a Chichinadze-like law and by assuming that temperature map is known. In the further work, we will focus on the development of a cost-effective alternative thermo-mechanical approach (coupling steady state mechanical analysis and transient thermal analysis) and work on the automation of the proposed approach and improve the implementation of this algorithm in ABAQUS. 


\section{References}

[1] O. A. Olatunbosum, E. O. Bolarinwa, "Finite element simulation of the tyre burst test", Proceedings of the Institution of Mechanical Engineers, Vol. 218, p. 1251-1258, (2006)

[2] M.H.R Ghoreish, "Finite Element Analysis of Steel-Belted Radial Tyre with Tread Pattern under Contact Load“, Iranian Polymer Journal, Vol.15 (8), p. 667-674, (2006)

[3] N. Lahellec, F. Mazerolle, J.C. Michel, Second-order estimate of the macroscopic behaviour of periodic hyperelastic composites: theory and experimental validation, Journal of the Mechanics and Physics of Solids, Vol. 52 , p. 27-49, (2004)

[4] Société de technologie Michelin. Le pneu - L'adhérence. Michelin France (2001)

[5] A. Kongo Kondé, I. Rosu, F. Lebon, O. Brardo, B. Devésa, Etude du comportement en roulement d'un pneu d'avion, Colloque Nationale de Calcul de Structure, Giens, France (2009)

[6] U. Nackenhorst, "The ALE-formulation of bodies in rolling contact - Theoritical foundations and finite element approach", Computational Methods in Applied Mechanics and Engineering, Vol.193, p. 4299-4322, (2004)

[7] N. Korunovic, M. Trajanovic, M. Stojkovic, Finite Element Model for Steady-state Rolling Tire Analysis, Journal of the Serbian Society for Computational Mechanics, vol. 1 (1), p. 63-79. (2007)

[8] H. Tuncay Yuksel, S. Karadeniz, A computation model to predict the thermomechanical behavior of automobile tires, Constitutive Models for Rubber III, Busfield et Muhr (2003).

[9] H. Sakai, K. Araki, Thermal Engineering Analysis of Rubber Vulcanization and Tread Temperatures during Severe Sliding of a Tire, Tire Science and Technology, Vol. 27 (1), p. 22-47, (1999)

[10] J. Awrejcewicz, Yu Pyr'yev, Nonsmooth Dynamics of Contacting Thermoelastic Bodies, Advances in Mechanics and Mathematics, Vol. 16, Springer (2009) 